\title{
Crystal structure of 2-(5-chloro-quinolin-8-yloxy)- $\mathrm{N}$-quinolin-8-yl-acetamide, $\mathrm{C}_{20} \mathrm{H}_{14} \mathrm{~N}_{3} \mathrm{O}_{2} \mathrm{Cl}$
}

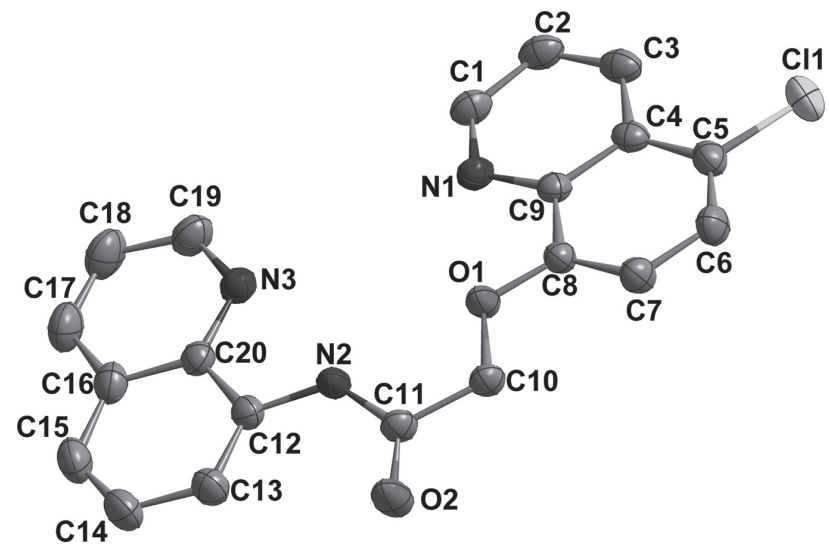

https://doi.org/10.1515/ncrs-2019-0907

Received December 12, 2019; accepted January 31, 2020; available online February 18, 2020

\section{Abstract \\ $\mathrm{C}_{20} \mathrm{H}_{14} \mathrm{~N}_{3} \mathrm{O}_{2} \mathrm{Cl}$, monoclinic, $P 2_{1} / \mathrm{c}$ (no. 14), $a=15.954(8) \AA$, $b=8.246(4) \AA, \quad c=14.383(7) \AA, \quad \beta=116.313(8)^{\circ}, \quad V=$ 1696.1(15) $\AA^{3}, \quad Z=4, \quad R_{\mathrm{gt}}(F)=0.0331, \quad w R_{\mathrm{ref}}\left(F^{2}\right)=0.0909$, $T=296 \mathrm{~K}$.}

\section{CCDC no.: 1981147}

The molecular structure is shown in the figure (hydrogen atoms are omitted for clarity). Table 1 contains crystallographic data and Table 2 contains the list of the atoms including atomic coordinates and displacement parameters.

\section{Source of material}

5-Chloro-8-hydroxyquinoline $(1.79 \mathrm{~g}, 10 \mathrm{mmol})$ and anhydrous potassium carbonate $(1.60 \mathrm{~g}, 11.6 \mathrm{mmol})$ were added to DMF (30 mL), then 2-chloro- $N$-quinolin-8-yl-acetamide $(2.20 \mathrm{~g}, 10.0 \mathrm{mmol})$ and a small quantity of KI were added. The reaction mixture was stirred for $5 \mathrm{~h}$ at $100-110^{\circ} \mathrm{C}$. After cooling, $150 \mathrm{~mL}$ water was added and stirred for $2 \mathrm{~h}$. The precipitate was collected by filtration and washed with water. Recrystallization from $\mathrm{CH}_{3} \mathrm{CN}$ gave colorless block crystals.

*Corresponding author: Wei-Na Wu, College of Chemistry and Chemical Engineering, Henan Polytechnic University, Jiaozuo 454000, P.R. China, e-mail: wuwnhpu@sina.com. https://orcid.org/0000-0002-0874-0703

Xiao-Hong Li: College of Chemistry and Chemical Engineering, Henan Polytechnic University, Jiaozuo 454000, P.R. China
Table 1: Data collection and handling.

\begin{tabular}{ll}
\hline Crystal: & Colorless block \\
Size: & $0.25 \times 0.18 \times 0.15 \mathrm{~mm}$ \\
Wavelength: & Mo $K \alpha$ radiation $(0.71073 \AA)$ \\
$\mu:$ & $0.25 \mathrm{~mm}^{-1}$ \\
Diffractometer, scan mode: & SMART, $\varphi$ and $\omega$ \\
$\theta_{\text {max }}$, completeness: & $25.0^{\circ}, 99 \%$ \\
$N(h k l)_{\text {measured }}, N(h k l)_{\text {unique }}, R_{\text {int }}:$ & $7607,2970,0.022$ \\
Criterion for $I_{\text {obs }}, N(h k l)_{\text {gt }}:$ & $I_{\text {obs }}>2 \sigma\left(l_{\text {obs }}\right), 2305$ \\
$N(\text { param })_{\text {refined }}:$ & 235 \\
Programs: & Bruker $[1]$, SHELX $[2,3]$ \\
\hline
\end{tabular}

Table 2: Fractional atomic coordinates and isotropic or equivalent isotropic displacement parameters $\left(\AA^{2}\right)$.

\begin{tabular}{lrrrr}
\hline Atom & $\boldsymbol{x}$ & $\boldsymbol{y}$ & $\boldsymbol{z}$ & $\boldsymbol{U}_{\text {iso }} / \boldsymbol{U}_{\text {eq }}$ \\
\hline Cl1 & $1.37482(3)$ & $0.59180(7)$ & $1.02415(5)$ & $0.0847(2)$ \\
O1 & $0.99629(7)$ & $0.35932(12)$ & $0.91121(9)$ & $0.0522(3)$ \\
O2 & $0.85161(8)$ & $0.02429(14)$ & $0.87801(11)$ & $0.0758(4)$ \\
N1 & $1.01771(9)$ & $0.64942(15)$ & $0.85088(10)$ & $0.0512(3)$ \\
N2 & $0.82200(8)$ & $0.28954(15)$ & $0.83867(10)$ & $0.0471(3)$ \\
H2A & 0.8493 & 0.3822 & 0.8485 & $0.057^{*}$ \\
N3 & $0.74690(10)$ & $0.56434(16)$ & $0.74687(11)$ & $0.0564(4)$ \\
C1 & $1.02877(13)$ & $0.7962(2)$ & $0.82335(13)$ & $0.0583(4)$ \\
H1B & 0.9753 & 0.8562 & 0.7842 & $0.070^{*}$ \\
C2 & $1.11432(14)$ & $0.8680(2)$ & $0.84840(14)$ & $0.0621(5)$ \\
H2B & 1.1176 & 0.9734 & 0.8272 & $0.075^{*}$ \\
C3 & $1.19299(13)$ & $0.7832(2)$ & $0.90388(14)$ & $0.0588(5)$ \\
H3B & 1.2512 & 0.8296 & 0.9215 & $0.071^{*}$ \\
C4 & $1.18649(11)$ & $0.62391(19)$ & $0.93503(12)$ & $0.0472(4)$ \\
C5 & $1.26297(11)$ & $0.5229(2)$ & $0.99161(13)$ & $0.0550(4)$ \\
C6 & $1.25081(12)$ & $0.3734(2)$ & $1.02066(14)$ & $0.0599(5)$ \\
H6A & 1.3027 & 0.3088 & 1.0582 & $0.072^{*}$ \\
C7 & $1.16193(12)$ & $0.3135(2)$ & $0.99557(13)$ & $0.0559(4)$ \\
H7A & 1.1548 & 0.2102 & 1.0170 & $0.067^{*}$ \\
C8 & $1.08570(10)$ & $0.40544(18)$ & $0.93997(12)$ & $0.0444(4)$ \\
C9 & $1.09599(10)$ & $0.56358(18)$ & $0.90752(11)$ & $0.0434(4)$ \\
C10 & $0.98043(11)$ & $0.19559(18)$ & $0.92655(13)$ & $0.0491(4)$ \\
H10A & 1.0076 & 0.1722 & 1.0002 & $0.059^{*}$ \\
H10B & 1.0101 & 0.1260 & 0.8955 & $0.059^{*}$ \\
C11 & $0.87781(11)$ & $0.16225(18)$ & $0.87853(12)$ & $0.0486(4)$ \\
C12 & $0.72521(11)$ & $0.29172(19)$ & $0.78317(12)$ & $0.0472(4)$ \\
C13 & $0.66768(12)$ & $0.1642(2)$ & $0.77282(14)$ & $0.0606(5)$ \\
H13A & 0.6925 & 0.0659 & 0.8049 & $0.073^{*}$ \\
C14 & $0.57134(13)$ & $0.1811(3)$ & $0.71406(17)$ & $0.0747(6)$ \\
H14A & 0.5327 & 0.0930 & 0.7073 & $0.090^{*}$ \\
C15 & $0.53291(13)$ & $0.3210(3)$ & $0.66713(17)$ & $0.0778(6)$ \\
H15A & 0.4684 & 0.3288 & 0.6286 & $0.093^{*}$ \\
C16 & $0.58979(12)$ & $0.4558(2)$ & $0.67592(14)$ & $0.0625(5)$ \\
& & & &
\end{tabular}


Table 2 (continued)

\begin{tabular}{lrrrr}
\hline Atom & $\boldsymbol{x}$ & $\boldsymbol{y}$ & $\boldsymbol{z}$ & $\boldsymbol{U}_{\text {iso }} \boldsymbol{U}_{\text {eq }}$ \\
\hline C17 & $0.55674(15)$ & $0.6065(3)$ & $0.62992(18)$ & $0.0836(6)$ \\
H17A & 0.4929 & 0.6222 & 0.5899 & $0.100^{*}$ \\
C18 & $0.61644(17)$ & $0.7285(3)$ & $0.64318(19)$ & $0.0873(7)$ \\
H18A & 0.5944 & 0.8292 & 0.6131 & $0.105^{\star}$ \\
C19 & $0.71158(14)$ & $0.7029(2)$ & $0.70247(16)$ & $0.0717(5)$ \\
H19A & 0.7523 & 0.7886 & 0.7110 & $0.086^{*}$ \\
C20 & $0.68685(11)$ & $0.4417(2)$ & $0.73451(12)$ & $0.0493(4)$ \\
\hline
\end{tabular}

\section{Experimental details}

The structure was solved by direct methods and refined with the SHELX crystallographic software package [3]. The hydrogen atoms were placed at calculated positions and refined as riding atoms with isotropic displacement parameters.

\section{Comment}

The quinoline-based amide compounds have attracted much attention mainly due to their excellent coordination ability [4-7] and high selectivity to metal ions [8].

There is one title molecule in the asymmetric unit (see the Figure). In the title compound, all bond lengths are comparable with those observed in a similar compound [7]. This is also true for the parent compoud [9] as well as for a related salt [10]. The dihedral angle between two quinoline ring moieties (N1/C1-C9, r.m.s. deviation 0.0142 Å; N3/C12-C20, r.m.s. deviation $0.0020 \AA$ ) is $7.8^{\circ}$. In the crystal structure, intramolecular $\mathrm{N}-\mathrm{H} \cdots \mathrm{O}$ hydrogen bonds between amide $\mathrm{N}$ and phenolic $\mathrm{O}$ atoms are present.

\section{References}

1. Bruker. SMART and SAINT. Bruker AXS Inc., Madison, WI, USA (2007).

2. Sheldrick, G. M.: SHELXT-integrated space-group and crystal-structure determination. Acta Crystallogr. A71 (2015) 3-8.

3. Sheldrick, G. M.: Crystal structure refinement with SHELX. Acta Crystallogr. C71 (2015) 3-8.

4. Cai, H.-X.; Wu, W.-N.; Wang, Y.: Synthesis and crystal structures of two copper(II) complexes containing amide type ligands. Chin. J. Inorg. Chem. 18 (2013) 845-849.

5. Lin, L.; Li, X.-H.; Zhang, B.; Zhang, Z.-Y.; Wu, W.-N.; Wang, Y.: Syntheses, crystal structures and fluorescence properties of $\mathrm{Cu}(\mathrm{II}) / \mathrm{Zn}(\mathrm{II}) / \mathrm{Ag}(\mathrm{I})$ complexes with an amide type ligand. Chin. J. Inorg. Chem. 32 (2016) 1653-1658.

6. Mao, P.-D.; Yan, L.-L.; Wu, W.-N.; Liu M.-Q.; Zhou, L.-H.; Fu, S.-L.: Lanthanide (La, Tb, Dy) complexes with quinolinyloxy acetamide ligand: crystal structures and fluorescence properties. Chin. J. Inorg. Chem. 32 (2016) 1095-1100.

7. Wang, M.-S.; Li, H.-Y.; Wu, W.-N.: Bis[N-benzyl-2-(quinolin-8yloxy)acetamide] monohydrate. Acta Crystallogr. E67 (2011) 01558.

8. Zhou, X.; Li, P.; Shi, Z.; Tang, X.; Chen, C.; Liu, W.: A highly selective fluorescent sensor for distinguishing cadmium from zinc ions based on a quinoline platform. Inorg. Chem. 51 (2012) 9226-9231.

9. Wen, Y.-H.; Xu, L.-L.; Bi, S.; Zhang, S.-S.: N-(Quinolin-8-yl)-2(quinolin-8-yl-oxy)acetamide. Acta Crystallogr. E62 (2006) $04476-04477$.

10. Kalita, D.; Baruah, J. B.: Different spatial orientations of amide derivatives on anion coordination. CrystEngComm 12 (2010) 1562-1567. 